\title{
More data on an exclusive group : Linux kernel developers and diversity (2005-2020)
}

\author{
Camille Akmut
}

February 5, 2020

abstract One hundred Linux kernel developers were reviewed, in total. Commits : $\geq 935$. (This group shares similar traits with early 20th c. college culture.)

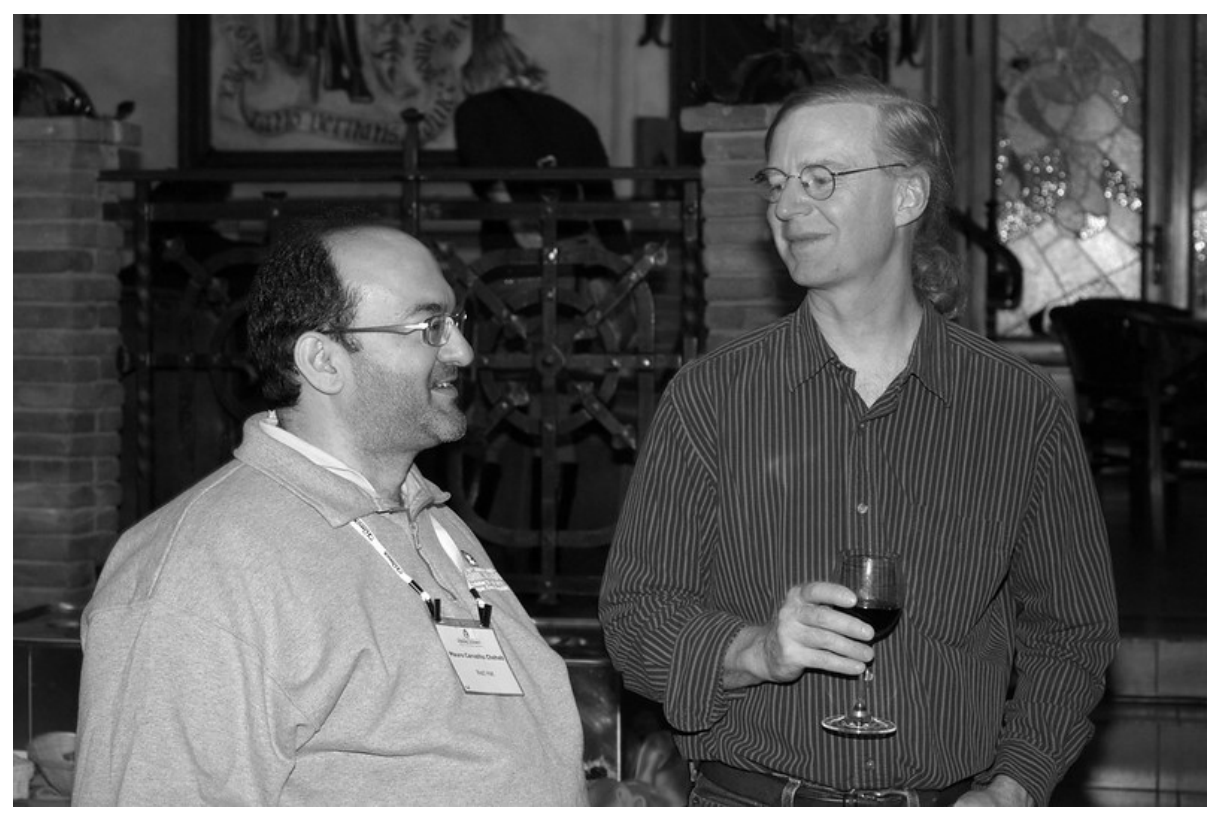

Figure 1: Linux kernel summit 2011 (Linux foundation) 
Table 1 - Diversity and Linux kernel developers, \#51-100 (2005-2020)

\begin{tabular}{|l|l|l|l|l|}
\hline$\ldots$ & & commits & & \\
\hline 51 & rostedt & 1,382 & Steven Rostedt & $\mathrm{m}$ \\
\hline 52 & geertu & 1,382 & Geert Uytterhoeven & $\mathrm{m}$ \\
\hline 53 & lwfinger & 1,362 & Larry.Finger (?) & $\mathrm{m}(?)$ \\
\hline 54 & alexandrebelloni & 1,354 & Alexandre Belloni & $\mathrm{m}$ \\
\hline 55 & JuliaLawall & 1,349 & Julia Lawall (?) & $\mathrm{f}(?)$ \\
\hline 56 & tomba & 1,340 & Tomi Valkeinen & $\mathrm{m}(?)$ \\
\hline 57 & olsajiri & 1,332 & Jiri Olsa (?) & $\mathrm{m}(?)$ \\
\hline 58 & bebarino & 1,315 & Stephen Boyd & $\mathrm{m}$ \\
\hline 59 & AlanStern & 1,300 & Alan Stern (?) & $\mathrm{m}(?)$ \\
\hline 60 & ujfalusi & 1,299 & Péter Ujfalusi & $\mathrm{m}$ \\
\hline 61 & ukleinek & 1,271 & Uwe Kleine-König & $\mathrm{m}$ \\
\hline 62 & wildea01 & 1,243 & Will Deacon (?) & $\mathrm{m}(?)$ \\
\hline 63 & jtlayton & 1,235 & Jeff Layton (?) & $\mathrm{m}$ \\
\hline 64 & dtor & 1,222 & Dmitry Torokhov & $\mathrm{m}$ \\
\hline 65 & palosaari & 1,206 & Antti Palosaari & $\mathrm{m}$ \\
\hline 66 & jnikula & 1,183 & Jani Nikula & $\mathrm{m}$ \\
\hline 67 & elfring & 1,170 & Markus Elfring & $\mathrm{m}$ \\
\hline 68 & egrumbach & 1,170 & Emmanuel Grumbach $(?)$ & $\mathrm{m}(?)$ \\
\hline 69 & paulgortmaker & 1,160 & Paul Gortmaker & $\mathrm{m}$ \\
\hline 70 & dwmw2 & 1,154 & David Woodhouse & $\mathrm{m}$ \\
\hline 71 & holtmann & 1,138 & Marcel Holtmann & $\mathrm{m}$ \\
\hline 72 & kuba-moo & 1,135 & Jakub Kicinski & $\mathrm{m}$ \\
\hline 73 & lag-linaro & 1,096 & Lee Jones & $\mathrm{m}$ \\
\hline 74 & jhedberg & 1,095 & Johan Hedberg & $\mathrm{m}$ \\
\hline 75 & paulusmack & 1,088 & Paul Mackerras & $\mathrm{m}$ \\
\hline 76 & rustyrussell & 1,083 & Rusty Russell & $\mathrm{m}$ \\
\hline 77 & martinezjavier & 1,057 & Javier Martinez Canillas & $\mathrm{m}$ \\
\hline 78 & bjorn-helgaas & 1,050 & Bjorn Helgaas & $\mathrm{m}$ \\
\hline 79 & rmilecki & 1,038 & Rafał Miłecki & $\mathrm{m}$ \\
\hline 80 & airlied & 1,035 & Dave Airlie & $\mathrm{m}$ \\
\hline 81 & borkmann & 1,027 & Daniel Borkmann & $\mathrm{m}$ \\
\hline 82 & fweisbec & 1,025 & Frederic Weisbecker & $\mathrm{m}$ \\
\hline 83 & akpm00 & 1,021 & Andrew Morton (?) & $\mathrm{m}(?)$ \\
\hline 84 & lyakh & 1,020 & Guennadi Liakhovetski & $\mathrm{m}$ \\
\hline 85 & jpirko & 1,018 & Jiri Pirko & $\mathrm{m}$ \\
\hline 86 & rjwysocki & 1,015 & Rafael J. Wysocki & $\mathrm{m}$ \\
\hline 87 & somasbw & 1,012 & Tomas Winkler & $\mathrm{m}$ \\
\hline 88 & 1,011 & Stephen Rothwell & $\mathrm{m}$ \\
\hline 89 & 1,008 & Oleg Nesterov (?) & $\mathrm{m}(?)$ \\
\hline 90 & 1,005 & David Sterba (?) & $?$ \\
\hline 91 & 999 & K. Y. Srinivasan (?) & \\
\hline & & & \\
\hline
\end{tabular}




\begin{tabular}{|l|l|l|l|l|}
\hline 92 & ardbiesheuvel & 998 & Ard Biesheuvel & $\mathrm{m}$ \\
\hline 93 & acpibob & 995 & Robert Moore & $\mathrm{m}$ \\
\hline 94 & dsahern & 982 & David Ahern & $\mathrm{m}$ \\
\hline 95 & tytso & 969 & Theodore Ts'o & $\mathrm{m}$ \\
\hline 96 & ebiggers & 968 & Eric Biggers & $\mathrm{m}$ \\
\hline 97 & michalsimek & 957 & Michal Simek & $\mathrm{m}$ \\
\hline 98 & wens & 955 & Chen-Yu Tsai & $\mathrm{m}$ \\
\hline 99 & aakoskin & 935 & Aaro Koskinen & $\mathrm{m}$ \\
\hline 100 & ummakynes & 931 & Pablo Neira Ayuso (?) & $\mathrm{m}(?)$ \\
\hline
\end{tabular}

Table 1: "...to master, excluding merge commits" /torvalds/linux/

Measured 05/02/20 c. 9.40 am (no refresh)

We continue to indicate information not directly derived from Github profiles with (?)

Table 2 - Aggregated data

\begin{tabular}{|l|l|l|}
\hline Gender & $\mathrm{n}$ & Percentage \\
\hline Female & 1 & $2 \%$ \\
\hline Male & 49 & $98 \%$ \\
\hline
\end{tabular}

Table 2: Diversity : Linux kernel developers, \#51-100 (2005-2020)

Table 3 - Total aggregated data

\begin{tabular}{|l|l|l|}
\hline Gender & $\mathrm{n}$ & Percentage \\
\hline Female & 1 & $1 \%$ \\
\hline Male & 99 & $99 \%$ \\
\hline
\end{tabular}

Table 3: Diversity, top 100 linux kernel developers (2005-2020) 


\section{References}

- "Linux kernel source tree". https://github.com/torvalds/linux/graphs/contributors

("Contributions to master, excluding merge commits")

- Linux Foundation photographies https://www.flickr.com/photos/linuxfoundation/22747710852/in/album-72157660376885370/

https://www.flickr.com/photos/linuxfoundation/6306036922/in/album-72157627910931679/

https://www.flickr.com/photos/linuxfoundation/6305975680/in/album-72157627910931679/

https://www.flickr.com/photos/linuxfoundation/5182564838/in/album-72157625402551490/

https://www.flickr.com/photos/linuxfoundation/30804670406/in/album-72157676268395315/

https://www.flickr.com/photos/linuxfoundation/30724804272/in/album-72157676268395315/

CC BY 2.0 (creativecommons.org)

\section{Addition to previous study}

Chris Wilson is ickle (based on "Signed-off-by" information in their commits)?

While we did our best, we may not have been able to identify all non-binary identities, just as binary ones. Sorry! 


\section{Annex}

- Linux kernel summit 2010 - group photo

- Linux kernel summit 2011 - group photo

- Linux kernel summit 2015 - group photo

- Linux kernel summit 2016 - group photo

- Linux kernel summit 2016 - gentlemen eating*

— Linux kernel summit 2011 - two gentlemen talk [front cover]

(*a woman is seen in the background) 